\title{
Erratum to: Matrix-assisted laser desorption/ionization imaging mass spectrometry revealed traces of dental problem associated with dental structure
}

\author{
Hirokazu Hirano • Noritaka Masaki • Takahiro Hayasaka • \\ Yoshiko Watanabe • Kazuma Masumoto • Tetsuji Nagata • \\ Fuminori Katou • Mitsutoshi Setou
}

Published online: 23 July 2013

(C) Springer-Verlag Berlin Heidelberg 2013

\section{Erratum to: Anal Bioanal Chem DOI: 10.1007/s00216-013-7075-y}

After online publication of the article the authors found the following typographical error in the Discussion section:

In the sentence "As $m / z$ 780.8, 782.9, and 810.7 were also detected as characteristic signals to hairy cell in organ of corti by IMS analysis [27], and taking account that the hairy cells are known to be similar to odontoblasts [28], which also sense mechanical stress at the periphery in dental pulp in the same manner, the odontoblasts in dental pulp were also expected to be detected in our present analysis" the description of " $m / z$ 780.8, 782.9, and 810.7" should be corrected to " $m / z$ 780.5, 782.5, and 810.5".

The online version of the original article can be found at http://dx.doi.org/ 10.1007/s00216-013-7075-y.

H. Hirano $\cdot$ Y. Watanabe $\cdot$ K. Masumoto $\cdot$ T. Nagata $\cdot$ F. Katou Department of Oral and Maxillofacial Surgery, Hamamatsu

University School of Medicine, 1-20-1 Handayama, Higashi-ku,

Hamamatsu, Shizuoka 431-3192, Japan

N. Masaki $\cdot$ T. Hayasaka $\cdot$ M. Setou $(\bowtie)$

Department of Cell Biology and Anatomy, Hamamatsu University

School of Medicine, 1-20-1 Handayama, Higashi-ku,

Hamamatsu, Shizuoka 431-3192, Japan

e-mail: setou@hama-med.ac.jp 